\title{
COVID-19
}

\section{Health Evidence Summary No.112}

\section{Kerry Millington \& Samantha Reddin}

Liverpool School of Tropical Medicine (LSTM) \& Institute of Development Studies 15 February 2021

This weekly COVID-19 health evidence summary (HES) is based on 3.5 hours of desk-based research. The summary is not intended to be a comprehensive summary of available evidence on COVID-19 but aims to make original documents easily accessible to decision makers which, if relevant to them, they should go to before making decisions.

\section{Epidemiology and modelling}

\begin{tabular}{|c|c|c|c|c|}
\hline $\begin{array}{l}\text { Publication } \\
\text { date }\end{array}$ & Title/URL & $\begin{array}{l}\text { Journal/Article } \\
\text { type }\end{array}$ & Summary & Keywords \\
\hline 11.02 .2021 & $\begin{array}{l}\text { Antibody } \\
\text { status and } \\
\text { incidence of } \\
\text { SARS-CoV- } \\
2 \text { infection } \\
\text { in health } \\
\text { care } \\
\text { workers }\end{array}$ & $\begin{array}{l}\text { NEJM | } \\
\text { Original } \\
\text { article }\end{array}$ & $\begin{array}{l}\text { - Investigation of incidence of } \\
\text { SARS-CoV-2 infection in } \\
12,541 \text { health care workers } \\
\text { in UK } \\
\text { - Presence of anti-spike or } \\
\text { anti-nucleocapsid IgG } \\
\text { antibodies was associated } \\
\text { with a substantially reduced } \\
\text { risk of SARS-CoV-2 } \\
\text { reinfection in the following } 6 \\
\text { months } \\
\end{array}$ & $\begin{array}{l}\text { Antibodies, } \\
\text { reinfection }\end{array}$ \\
\hline 03.02.2021 & $\begin{array}{l}\text { Recurrent } \\
\text { deletions in } \\
\text { the SARS- } \\
\text { CoV-2 } \\
\text { spike } \\
\text { glycoprotein } \\
\text { drive } \\
\text { antibody } \\
\text { escape }\end{array}$ & $\begin{array}{l}\text { Science | } \\
\text { Report }\end{array}$ & $\begin{array}{l}\text { - Coronaviruses acquire } \\
\text { substitutions more slowly } \\
\text { than other RNA viruses, due } \\
\text { to a proofreading } \\
\text { polymerase } \\
\text { - In the spike glycoprotein, } \\
\text { recurrent deletions are } \\
\text { found that overcome this } \\
\text { slow substitution rate } \\
\text { Deletion variants arise in } \\
\text { diverse genetic and } \\
\text { geographic backgrounds } \\
\text { and can transmit efficiently } \\
\text { Deletions frequently in the } \\
\text { recurrent deletion regions } \\
\text { (RDRs) confer resistance to } \\
\text { neutralising antibodies }\end{array}$ & $\begin{array}{l}\text { Variants, } \\
\text { immune } \\
\text { escape }\end{array}$ \\
\hline
\end{tabular}




\begin{tabular}{|l|l|l|} 
& $\begin{array}{l}\text { - } \\
\text { in immunocompromised } \\
\text { imatients remain to be } \\
\text { resolved } \\
\text { recurrence of adaptations } \\
\text { in single patients, and on } \\
\text { global scales highlights the } \\
\text { importance of tracking and } \\
\text { monitoring deletion variants }\end{array}$ \\
\hline
\end{tabular}

\section{Therapeutics}

\begin{tabular}{|c|c|c|c|c|}
\hline $\begin{array}{l}\text { Publication } \\
\text { date }\end{array}$ & Title/URL & $\begin{array}{l}\text { Journal/Articl } \\
\text { e type }\end{array}$ & Summary & Keywords \\
\hline 12.02.2021 & $\begin{array}{l}\text { Chloroquine or } \\
\text { hydroxychloroq } \\
\text { uine for } \\
\text { prevention and } \\
\text { treatment of } \\
\text { COVID-19 }\end{array}$ & $\begin{array}{l}\text { Cochrane | } \\
\text { Systematic } \\
\text { Review }\end{array}$ & $\begin{array}{l}\text { - SR of } \\
\text { randomised } \\
\text { controlled trials to } \\
\text { evaluate the } \\
\text { effects of } \\
\text { chloroquine o } \\
\text { hydroxychloroqui } \\
\text { ne (HCQ) in } 3 \\
\text { subgroups: (1) } \\
\text { treating people } \\
\text { with C19 on } \\
\text { death and time to } \\
\text { clearance of the } \\
\text { virus; (2) } \\
\text { preventing } \\
\text { infection in } \\
\text { people at risk of } \\
\text { SARS-CoV-2 } \\
\text { exposure; (3) } \\
\text { preventing } \\
\text { infection in } \\
\text { people exposed } \\
\text { to SARS-CoV-2 } \\
\text { HCQ for people } \\
\text { infected with } \\
\text { COVID-19 has } \\
\text { little or no effect } \\
\text { on the risk of } \\
\text { death and } \\
\text { probably no } \\
\text { effect on } \\
\text { progression to } \\
\text { mechanical } \\
\text { ventilation } \\
\text { There should be } \\
\text { no further trials of } \\
\text { HCQ or }\end{array}$ & $\begin{array}{l}\text { Chloroquine, } \\
\text { hydroxychloroquin } \\
\text { e }\end{array}$ \\
\hline
\end{tabular}




\begin{tabular}{|c|c|c|c|c|c|}
\hline & & & & $\begin{array}{l}\text { chloroquine for } \\
\text { treatment of } \\
\text { COVID-19 } \\
\text { Given these } \\
\text { results, it is } \\
\text { unlikely that the } \\
\text { drug is effective } \\
\text { in protecting } \\
\text { people from } \\
\text { infection, } \\
\text { although this is } \\
\text { not excluded, and } \\
\text { high-quality trials } \\
\text { examining } \\
\text { prevention of } \\
\text { infection could be } \\
\text { carried out to } \\
\text { give a clear } \\
\text { answer }\end{array}$ & \\
\hline 11.02 .2021 & $\begin{array}{l}\text { Tocilizumab in } \\
\text { patients } \\
\text { admitted to } \\
\text { hospital with } \\
\text { COVID-19 } \\
\text { (RECOVERY): } \\
\text { preliminary } \\
\text { results of a } \\
\text { randomised, } \\
\text { controlled, } \\
\text { open-label, } \\
\text { platform trial }\end{array}$ & $\begin{array}{l}\text { medRxiv | } \\
\text { pre-print (not } \\
\text { peer } \\
\text { reviewed) }\end{array}$ & & $\begin{array}{l}\text { Randomised, } \\
\text { controlled, open- } \\
\text { label, platform } \\
\text { trial in } 4116 \text { adult } \\
\text { patients with } \\
\text { hypoxia (oxygen } \\
\text { saturation <92\% } \\
\text { on air or requiring } \\
\text { oxygen therapy) } \\
\text { and evidence of } \\
\text { systemic } \\
\text { inflammation (C- } \\
\text { reactive protein } \\
>=75 m g / L \text { ) } \\
\text { randomised to } \\
\text { usual standard of } \\
\text { care alone versus } \\
\text { usual standard of } \\
\text { care plus } \\
\text { tocilizumab at a } \\
\text { dose of } 400 \text { mg } \\
\text { to } 800 \text { mg } \\
\text { (depending on } \\
\text { weight) given } \\
\text { intravenously } \\
\text { Primary outcome } \\
\text { was } 28-d a y \\
\text { mortality, } \\
\text { assessed in the } \\
\text { intention-to-treat } \\
\text { population } \\
\text { Findings are that } \\
\text { in hospitalised } \\
\text { covID-19 }\end{array}$ & tocilizumab \\
\hline
\end{tabular}




\begin{tabular}{|c|c|c|c|c|c|}
\hline & & & & $\begin{array}{l}\text { patients with } \\
\text { hypoxia and } \\
\text { systemic } \\
\text { inflammation, } \\
\text { tocilizumab } \\
\text { improved survival } \\
\text { and other clinical } \\
\text { outcomes } \\
\text { Benefits seen } \\
\text { irrespective of the } \\
\text { level of } \\
\text { respiratory } \\
\text { support and were } \\
\text { additional to the } \\
\text { benefits of } \\
\text { systemic } \\
\text { corticosteroids }\end{array}$ & \\
\hline 08.02.2021 & $\begin{array}{l}\text { Inhaled } \\
\text { budesonide in } \\
\text { the treatment of } \\
\text { early COVID-19 } \\
\text { illness: a } \\
\text { randomised } \\
\text { controlled trial }\end{array}$ & $\begin{array}{l}\text { medRxiv | } \\
\text { pre-print (not } \\
\text { peer } \\
\text { reviewed) }\end{array}$ & & $\begin{array}{l}\text { Early in the } \\
\text { pandemic, } \\
\text { observed that } \\
\text { patients with } \\
\text { chronic } \\
\text { respiratory } \\
\text { disease, who are } \\
\text { often prescribed } \\
\text { inhaled steroids, } \\
\text { were significantly } \\
\text { under- } \\
\text { represented } \\
\text { among those } \\
\text { admitted to } \\
\text { hospital with } \\
\text { COVID-19 } \\
\text { Phase } 2 \\
\text { randomised, } \\
\text { open label trial of } \\
\text { inhaled } \\
\text { budesonide } \\
\text { versus usual care } \\
\text { in } 146 \text { adults } \\
\text { within } 7 \text { days of } \\
\text { onset of mild } \\
\text { Covid-19 } \\
\text { symptoms } \\
\text { (STOIC trial) } \\
\text { Treatment with } \\
\text { inhaled } \\
\text { budesonide } \\
\text { (corticosteroid } \\
\text { used in long-term } \\
\text { management of } \\
\text { asthma and } \\
\text { cOPD) given to }\end{array}$ & budesonide \\
\hline
\end{tabular}




$\mid$\begin{tabular}{|l|l|}
$\mid$ & patients with \\
& COVID-19 within \\
seven days of \\
symptom onset \\
reduced the need \\
& for urgent care, \\
& hospitalisation \\
& and recovery \\
& time in people \\
& with COVID-19 \\
\hline
\end{tabular}

\section{Vaccines}

\begin{tabular}{|c|c|c|c|c|}
\hline $\begin{array}{l}\text { Publication } \\
\text { date }\end{array}$ & Title/URL & $\begin{array}{l}\text { Journal/Article } \\
\text { type }\end{array}$ & Summary & Keywords \\
\hline 12.02 .2021 & $\begin{array}{l}\text { Challenges } \\
\text { in ensuring } \\
\text { global } \\
\text { access to } \\
\text { COVID-19 } \\
\text { vaccines: } \\
\text { production, } \\
\text { affordability, } \\
\text { allocation, } \\
\text { and } \\
\text { deployment }\end{array}$ & $\begin{array}{l}\text { The Lancet | } \\
\text { Health Policy }\end{array}$ & $\begin{array}{l}\text { Review of potential } \\
\text { challenges to success in of } \\
\text { COVID-19 production, } \\
\text { affordability, allocations and } \\
\text { deployment and a } \\
\text { discussion of policy } \\
\text { implications } \\
\text { - Includes a dashboard to } \\
\text { highlight key characteristics } \\
\text { of } 26 \text { leading vaccine } \\
\text { candidates with a traffic- } \\
\text { light system to signal the } \\
\text { potential contributions of } \\
\text { each candidate in achieving } \\
\text { global vaccine immunity } \\
\text { and important trade-offs in } \\
\text { policy and implementation } \\
\text { Also } 32-\text { country survey from } \\
\text { Oct to Dec } 2020 \text { on } \\
\text { potential acceptance of } \\
\text { COVID-19 vaccines }\end{array}$ & $\begin{array}{l}\text { COVID-19 } \\
\text { vaccines, } \\
\text { global } \\
\text { access }\end{array}$ \\
\hline 11.02 .2021 & $\begin{array}{l}\text { A Covid } \\
\text { Vaccine } \\
\text { Certificate: } \\
\text { building on } \\
\text { lessons } \\
\text { from digital } \\
\text { ID for the } \\
\text { digital } \\
\text { yellow card }\end{array}$ & CGD | Notes & $\begin{array}{l}\text { - A Covid Vaccine Certificate } \\
\text { (CVC) is likely to become } \\
\text { an important tool to help } \\
\text { monitor and manage the } \\
\text { rollout of vaccinations and } \\
\text { in the recovery of national } \\
\text { economies, facilitate safe } \\
\text { movement of people across } \\
\text { countries and rejuvenate } \\
\text { tourism, important for many } \\
\text { developing countries } \\
\text { These notes consider some } \\
\text { lessons from rollout of } \\
\text { identification systems } \\
\text { across the world }\end{array}$ & $\begin{array}{l}\text { Covid } \\
\text { vaccine } \\
\text { certificate }\end{array}$ \\
\hline
\end{tabular}




\begin{tabular}{|c|c|c|c|c|}
\hline & & & $\begin{array}{l}\text { - Low-cost, high-access } \\
\text { approaches are essential to } \\
\text { ensure that the digital and } \\
\text { affordability gap does not } \\
\text { widen, balancing Level of } \\
\text { Assurance against Level of } \\
\text { Security }\end{array}$ & \\
\hline 10.02 .2021 & $\begin{array}{l}\text { mRNA } \\
\text { vaccine- } \\
\text { elicited } \\
\text { antibodies } \\
\text { to SARS- } \\
\text { CoV-2 and } \\
\text { circulating } \\
\text { variants }\end{array}$ & $\begin{array}{l}\text { Nature | } \\
\text { Accelerated } \\
\text { Article } \\
\text { Preview }\end{array}$ & $\begin{array}{l}\text { - A report on antibody and } \\
\text { memory B cell responses in } \\
20 \text { volunteers eight weeks } \\
\text { after second vaccination } \\
\text { with either Moderna or } \\
\text { Pfizer-BioNTec vaccines } \\
\text { - Modest effects of the } \\
\text { mutations on viral sensitivity } \\
\text { to plasma reflects } \\
\text { polyclonal nature of } \\
\text { neutralising antibodies } \\
\text { Mutations maybe emerging } \\
\text { in response to immune } \\
\text { selection in individuals with } \\
\text { non-sterilising immunity } \\
\text { Plasma neutralising activity, } \\
\text { and relative numbers of } \\
\text { receptor binding domain- } \\
\text { specific memory B cells } \\
\text { were equivalent to those } \\
\text { recovering from natural } \\
\text { infection } \\
\text { Authors conclude that } \\
\text { monoclonal antibodies in } \\
\text { clinical use should be } \\
\text { tested against newly arising } \\
\text { variants and that mRNA } \\
\text { vaccines may need to be } \\
\text { updated periodically to } \\
\text { avoid loss of clinical } \\
\text { efficacy }\end{array}$ & $\begin{array}{l}\text { Immunity, } \\
\text { mRNA } \\
\text { vaccines }\end{array}$ \\
\hline
\end{tabular}

\section{Indirect impact of COVID-19}

\begin{tabular}{|l|l|l|l|l|}
\hline $\begin{array}{l}\text { Publication } \\
\text { date }\end{array}$ & Title/URL & $\begin{array}{l}\text { Journal/Article } \\
\text { type }\end{array}$ & Summary & Keywords \\
\hline Jan 2021 & $\begin{array}{l}\text { Impact of } \\
\text { Covid-19 on } \\
\text { adolescent } \\
\text { mental } \\
\text { health in } \\
\text { Viet Nam }\end{array}$ & $\begin{array}{l}\text { ODI | Working } \\
\text { and } \\
\text { discussion } \\
\text { paper }\end{array}$ & $\begin{array}{l}\text { A literature review of the } \\
\text { impacts of COVID-19 on } \\
\text { mental well-being and the } \\
\text { mental health and } \\
\text { psychosocial support } \\
\text { needs of adolescents in } \\
\text { Viet Nam and Tanzania } \\
\text { COVID-19 has led to an } \\
\text { increase in mental health }\end{array}$ & $\begin{array}{l}\text { Adolescent } \\
\text { mental } \\
\text { health, Viet }\end{array}$ \\
Tam, \\
Tanzania
\end{tabular}




\begin{tabular}{|c|c|}
\hline $\begin{array}{l}\text { and } \\
\text { Tanzania }\end{array}$ & $\begin{array}{l}\text { disorder, particularly } \\
\text { anxiety, stress and } \\
\text { depressive disorders with a } \\
\text { particular impact from } \\
\text { school closures and online } \\
\text { learning } \\
\text { - Older adolescents stress } \\
\text { about examinations, their } \\
\text { uncertain future and job } \\
\text { prospects } \\
\text { Increased provision in } \\
\text { services dedicated to } \\
\text { combating domestic and } \\
\text { online violence given } \\
\text { increased exposure to } \\
\text { abusive household } \\
\text { conditions and online child } \\
\text { abuse material } \\
\text { A variety of digital } \\
\text { interventions were } \\
\text { introduced in both } \\
\text { countries, in response to } \\
\text { restrictions on providing in- } \\
\text { person mental health } \\
\text { services, reaching millions } \\
\text { of adolescents globally. } \\
\text { Key lessons must be learnt } \\
\text { from this for post-Covid } \\
\text { engagement and particular } \\
\text { attention to accessibility } \\
\end{array}$ \\
\hline
\end{tabular}

\section{Comments, Editorials, Opinions, Blogs, News}

\begin{tabular}{|l|l|l|}
\hline $\begin{array}{l}\text { Publication } \\
\text { date }\end{array}$ & Title/URL & Journal | Article type \\
\hline 15.02.2021 & $\begin{array}{l}\text { Covid-19: are cloth masks still effective? And other } \\
\text { questions answered }\end{array}$ & BMJ | News analysis \\
\hline 13.02.2021 & Tanzania refuses COVID-19 vaccines & $\begin{array}{l}\text { The Lancet | World } \\
\text { Report }\end{array}$ \\
\hline 12.02.2021 & $\begin{array}{l}\text { How 'killer' T cells could boost COVID immunity in face } \\
\text { of new variants }\end{array}$ & Nature | News \\
\hline 12.02.2021 & $\begin{array}{l}\text { Genomic-informed pathogen surveillance in Africa: } \\
\text { opportunities and challenges }\end{array}$ & $\begin{array}{l}\text { The Lancet Infectious } \\
\text { Diseases | Personal view }\end{array}$ \\
\hline
\end{tabular}




\begin{tabular}{|c|c|c|}
\hline 12.02 .2021 & $\begin{array}{l}\text { Oxford University extends COVID-19 vaccine study to } \\
\text { children }\end{array}$ & $\begin{array}{l}\text { University of Oxford I } \\
\text { News }\end{array}$ \\
\hline 12.02 .2021 & $\begin{array}{l}\text { Can Covax deliver the vaccines much of the world } \\
\text { needs? }\end{array}$ & Financial Times | News \\
\hline 12.02 .2021 & $\begin{array}{l}\text { Priorities for the COVID-19 pandemic at the start of } \\
\text { 2021: statement of the Lancet COVID-19 Commission }\end{array}$ & The Lancet | Comment \\
\hline 12.02 .2021 & $\begin{array}{l}\text { The hazards of medicine by tweet: the case of anti- } \\
\text { cytokine therapy for Covid-19 }\end{array}$ & Statnews \\
\hline 11.02 .2021 & The COVID-19 exit strategy - why we need to aim low & $\begin{array}{l}\text { The Lancet Infectious } \\
\text { Diseases | Editorial }\end{array}$ \\
\hline 11.02 .2021 & $\begin{array}{l}\text { SARS-CoV-2 variants and ending the COVID-19 } \\
\text { pandemic }\end{array}$ & The Lancet | Comment \\
\hline 11.02 .2021 & $\begin{array}{l}\text { SARS-CoV-2 variants and ending the COVID-19 } \\
\text { pandemic }\end{array}$ & The Lancet | Comment \\
\hline 10.02 .2021 & Redesigning health systems for global health security & $\begin{array}{l}\text { The Lancet Global } \\
\text { Health | Comment }\end{array}$ \\
\hline 10.02 .2021 & Oxygen supplies and COVID-19 mortality in Africa & $\begin{array}{l}\text { The Lancet Respiratory } \\
\text { Medicine | News }\end{array}$ \\
\hline 10.02 .2021 & $\begin{array}{l}\text { Drug trial that could improve respiratory recovery from } \\
\text { COVID-19 now underway }\end{array}$ & $\begin{array}{l}\text { University of Oxford | } \\
\text { News }\end{array}$ \\
\hline 10.02 .2021 & $\begin{array}{l}\text { The rich people's virus? Latest reflection from } \\
\text { Zimbabwe }\end{array}$ & SSHAP | Blog \\
\hline 10.02 .2021 & $\begin{array}{l}\text { Expanding health product manufacturing in Africa: } \\
\text { ideas for development finance institutions, procurers, } \\
\text { and policymakers }\end{array}$ & CGD | Blog \\
\hline 10.02 .2021 & Release COVID-19 vaccine contracts & CGD | Blog \\
\hline
\end{tabular}




\begin{tabular}{|l|l|l|} 
10.02.2021 & $\begin{array}{l}\text { How will COVID-19 impact our progress towards } \\
\text { Universal Health Coverage }\end{array}$ & CGD | Blog \\
\hline 09.02.2021 & Rapid coronavirus tests: a guide for the perplexed & Nature | News \\
\hline 09.02.2021 & $\begin{array}{l}\text { We can stop preventable COVID deaths by urgently } \\
\text { prioritising medical oxygen and essential critical care }\end{array}$ & CGD | Blog \\
\hline 01.02.2021 & $\begin{array}{l}\text { COVID crisis is fueling food price rises for world's } \\
\text { poorest }\end{array}$ & World Bank | Blog \\
\hline 07.01.2021 & $\begin{array}{l}\text { What COVID-19 can mean for long-term inequality in } \\
\text { developing countries }\end{array}$ & World Bank | Blog \\
\hline 03.02.2021 & $\begin{array}{l}\text { Put to the test: use of rapid testing technologies for } \\
\text { covid-19 }\end{array}$ & BMJ | analysis \\
\hline
\end{tabular}

\section{Guidelines, Statements \& Tools}

\begin{tabular}{|c|c|c|c|}
\hline $\begin{array}{l}\text { Publication } \\
\text { Date }\end{array}$ & Title/URL & Source & Summary \\
\hline 10.02 .2021 & $\begin{array}{l}\text { Interim } \\
\text { recommendations } \\
\text { for use of the } \\
\text { AZD1222 } \\
\text { (ChAdOx1-S } \\
\text { (recombinant)) } \\
\text { vaccine against } \\
\text { COVID-10 } \\
\text { developed by Oxford } \\
\text { University and } \\
\text { AstraZeneca }\end{array}$ & $\begin{array}{l}\text { WHO I } \\
\text { Interim } \\
\text { guidelines }\end{array}$ & $\begin{array}{l}\text { - Interim guidance developed on } \\
\text { the basis of WHO SAGE advice } \\
\text { Recommendation is two standard } \\
\text { doses of ChAdOx1 nCoV-19 } \\
\text { administered at an 8-12 week } \\
\text { interval in people aged } 18 \text { years } \\
\text { and older } \\
\text { - Priority to be given to health } \\
\text { workers at high risk of exposure } \\
\text { and older people, including those } \\
\text { aged } 65 \text { or older } \\
\text { - Further studies are required for } \\
\text { persons living with HIV or auto- } \\
\text { immune or immunocompromised } \\
\text { but may be vaccinated after } \\
\text { receiving information and } \\
\text { counselling }\end{array}$ \\
\hline Jan 2021 & The inequality virus & $\begin{array}{l}\text { Oxfam | } \\
\text { Report }\end{array}$ & $\begin{array}{l}\text { Documenting how COVID-19 has } \\
\text { affected different populations and } \\
\text { parts of the world and impacts on } \\
\text { those already-marginalised }\end{array}$ \\
\hline
\end{tabular}




\section{Dashboards \& Trackers}

\begin{tabular}{|c|c|c|c|c|c|c|c|}
\hline $\begin{array}{l}\text { Cases \& deaths: } \\
\text { Global }\end{array}$ & $\begin{array}{l}\text { Cases \& } \\
\text { deaths: } \\
\text { Regional }\end{array}$ & $\begin{array}{l}\text { Cases \& } \quad \& \\
\text { deaths: } \\
\text { Country }\end{array}$ & $\begin{array}{l}\text { Living evidence \& } \\
\text { policy maps }\end{array}$ & $\begin{array}{l}\text { Current research } \\
\text { including trials }\end{array}$ & Diagnostics & Treatments & Vaccines \\
\hline WHO sitreps & WHO Africa & Ghana & COVID-NMA & WHO & $\begin{array}{l}\text { FIND SARS- } \\
\text { CoV-2 Test } \\
\text { Tracker }\end{array}$ & $\begin{array}{l}\text { Global COVID- } \\
19 \text { Clinical Trial } \\
\text { Tracker }\end{array}$ & CEPI \\
\hline WHO dashboard & $\begin{array}{l}\text { African } \\
\text { Arguments }\end{array}$ & Indonesia & EPPI Centre & $\begin{array}{l}\text { WHO International } \\
\text { Clinical Trials } \\
\text { Registry Platform } \\
\text { (ICTRP) }\end{array}$ & $\begin{array}{l}\text { FIND SARS- } \\
\text { CoV-2 } \\
\text { Diagnostics: } \\
\text { performance } \\
\text { data }\end{array}$ & $\begin{array}{l}\text { US NIH } \\
\text { registered } \\
\text { clinical trials }\end{array}$ & $\begin{array}{l}\text { Vaccine Centre } \\
\text { LSHTM }\end{array}$ \\
\hline $\begin{array}{l}\text { Johns Hopkins } \\
\text { University }\end{array}$ & $\begin{array}{l}\text { European } \\
\text { CDC }\end{array}$ & Nigeria CDC & $\begin{array}{l}\text { Norwegian } \\
\text { Institute of Public } \\
\text { Health }\end{array}$ & Cytel & $\begin{array}{l}\text { Serology-based } \\
\text { tests for COVID- } \\
19\end{array}$ & Solidarity trial & $\begin{array}{l}\text { COVID-19 } \\
\text { Oxford Vaccine } \\
\text { Trial }\end{array}$ \\
\hline WEF & & Sierra Leone & $\begin{array}{l}\text { Oxford C19 } \\
\text { Government } \\
\text { Response } \\
\text { Tracker } \\
\text { (OxCGRT) }\end{array}$ & US NIH & $\begin{array}{l}\text { Our World in } \\
\text { Data: C19 } \\
\text { Testing }\end{array}$ & $\begin{array}{l}\text { COVID-19 } \\
\text { Therapeutics } \\
\text { Accelerator }\end{array}$ & $\begin{array}{l}\text { COVID-19 } \\
\text { Vaccine Tracker }\end{array}$ \\
\hline
\end{tabular}




\begin{tabular}{|c|c|c|c|c|}
\hline $\begin{array}{l}\text { Our World in } \\
\text { Data }\end{array}$ & Singapore & $\begin{array}{l}\text { Our World in } \\
\text { Data: C19 Policy } \\
\text { responses }\end{array}$ & COVID-evidence & $\begin{array}{l}\text { Our World in } \\
\text { Data: COVID- } \\
19 \text { vaccinations }\end{array}$ \\
\hline Global 5050 & UK & $\begin{array}{l}\text { IFPRI COVID-19 } \\
\text { Policy Response } \\
\text { Portal }\end{array}$ & Cochrane & \\
\hline $\begin{array}{l}\text { CEBM, } \\
\text { University of } \\
\text { Oxford }\end{array}$ & US & $\begin{array}{l}\text { COVID-19 } \\
\text { Primer }\end{array}$ & Clinicaltrials.gov & \\
\hline $\begin{array}{l}\text { Humanitarian } \\
\text { Data Exchange }\end{array}$ & & NIH LitCovid & UKCDR & \\
\hline $\begin{array}{l}\text { Information is } \\
\text { Beautiful }\end{array}$ & & $\begin{array}{l}\text { WHO COVID-19 } \\
\text { Database }\end{array}$ & & \\
\hline LSHTM & & & & \\
\hline $\begin{array}{l}\text { HealthMap } \\
\text { (cases) }\end{array}$ & & & & \\
\hline $\begin{array}{l}\text { The Commons } \\
\text { Project }\end{array}$ & & & & \\
\hline SeroTracker & & & & \\
\hline
\end{tabular}




\section{C19 Resource Hubs}

\begin{tabular}{|c|c|c|c|c|c|}
\hline Global & $\begin{array}{l}\text { Regional } \\
\text { \& Country }\end{array}$ & $\begin{array}{l}\text { Academic } \\
\text { journals \& } \\
\text { Publishers }\end{array}$ & $\begin{array}{l}\text { Institutes/Centres/ } \\
\text { Funders/Other }\end{array}$ & $\begin{array}{l}\text { Health } \\
\text { Topics }\end{array}$ & $\begin{array}{l}\text { Social } \\
\text { Sciences }\end{array}$ \\
\hline $\begin{array}{l}\text { WHO } \\
\text { COVID-19 } \\
\text { pandemic }\end{array}$ & $\begin{array}{l}\text { Africa } \\
\text { CDC }\end{array}$ & $\begin{array}{l}\text { Annals of } \\
\text { Internal Medicine }\end{array}$ & LSTM & $\begin{array}{l}\text { Stop TB } \\
\text { Partnership }\end{array}$ & SSHAP \\
\hline $\begin{array}{l}\text { WHO risk } \\
\text { communicati } \\
\text { on }\end{array}$ & $\begin{array}{l}\text { African } \\
\text { Union }\end{array}$ & BMJ & LSHTM & & IDA \\
\hline WHO Q\&A & $\begin{array}{l}\text { Nigeria } \\
\text { CDC }\end{array}$ & $\begin{array}{l}\text { Bulletin of the } \\
\text { WHO }\end{array}$ & $\begin{array}{l}\text { ICL MRC Centre } \\
\text { for Global } \\
\text { Infectious Disease } \\
\text { Analysis }\end{array}$ & $\begin{array}{l}\text { Global } \\
\text { Menstrual } \\
\text { Collective }\end{array}$ & $\begin{array}{l}\text { Disability } \\
\text { and } \\
\text { inclusion }\end{array}$ \\
\hline $\begin{array}{l}\text { WHO Global } \\
\text { research }\end{array}$ & $\begin{array}{l}\text { GeoPoll: } \\
\text { SSA }\end{array}$ & $\begin{array}{l}\text { Cambridge } \\
\text { University Press }\end{array}$ & ODI & $\begin{array}{l}\text { SLH: } \\
\text { Handwashin } \\
\mathrm{g} \text { in low } \\
\text { resource } \\
\text { settings }\end{array}$ & $\begin{array}{l}\text { Coregrou } \\
\text { p IDDC }\end{array}$ \\
\hline $\begin{array}{l}\text { COVID-19 } \\
\text { Solidarity } \\
\text { Response } \\
\text { Fund }\end{array}$ & $\begin{array}{l}\text { Global } \\
\text { Health } \\
\text { Network } \\
\text { Africa }\end{array}$ & Cell Press & $\begin{array}{l}\text { Johns Hopkins } \\
\text { University }\end{array}$ & $\begin{array}{l}\text { RBM } \\
\text { Partnership }\end{array}$ & $\begin{array}{l}\text { Ethics, } \\
\text { health } \\
\text { systems \& } \\
\text { COVID-19 }\end{array}$ \\
\hline UN & $\begin{array}{l}\text { African } \\
\text { Academy } \\
\text { of } \\
\text { Sciences }\end{array}$ & Cochrane & $\begin{array}{l}\text { Center for Global } \\
\text { Development }\end{array}$ & $\begin{array}{l}\text { Epidemic } \\
\text { Preparednes } \\
\text { S } \\
\text { Innovations }\end{array}$ & $\begin{array}{l}\text { Social } \\
\text { Developm } \\
\text { ent Direct } \\
\text { C19 blog } \\
\text { series }\end{array}$ \\
\hline UN Women & $\begin{array}{l}\text { Africa } \\
\text { Evidence } \\
\text { Network }\end{array}$ & Elsevier & $\begin{array}{l}\text { CMMID } \\
\text { Repository }\end{array}$ & & \\
\hline
\end{tabular}




\begin{tabular}{|c|c|c|c|c|}
\hline UNOCHA & $\begin{array}{l}\text { OCHA } \\
\text { Southern } \\
\text { and } \\
\text { Eastern } \\
\text { Africa } \\
\text { COVID-19 } \\
\text { Digest }\end{array}$ & $\begin{array}{l}\text { Health Policy } \\
\text { and Planning }\end{array}$ & $\begin{array}{l}\text { Norwegian } \\
\text { Institute of Public } \\
\text { Health }\end{array}$ & \\
\hline UNHCR & $\begin{array}{l}\text { South } \\
\text { African } \\
\text { Governme } \\
\text { nt }\end{array}$ & JAMA Network & $\begin{array}{l}\text { Oxford Centre for } \\
\text { Evidence-based } \\
\text { Medicine }\end{array}$ & \\
\hline UNICEF & & The Lancet & HEART & \\
\hline UNESCO & & $\begin{array}{l}\text { medRxiv and } \\
\text { bioRxiv } \\
\text { (Preprints) }\end{array}$ & UKRI & \\
\hline UN WFP & & NEJM & Evidence Aid & \\
\hline GOARN & & $\begin{array}{l}\text { Oxford University } \\
\text { Press }\end{array}$ & $\mathrm{NIH}$ & \\
\hline EPI-WIN & & PLoS & $\begin{array}{l}\text { IFPRI Resources } \\
\text { and Analyses of } \\
\text { C19 Impact }\end{array}$ & \\
\hline World Bank & & SAGE journals & $\begin{array}{l}\text { Prevent } \\
\text { Epidemics }\end{array}$ & \\
\hline $\begin{array}{l}\text { Our World in } \\
\text { Data }\end{array}$ & & Science & & \\
\hline $\begin{array}{l}\text { COVID-19 } \\
\text { Narratives by } \\
\text { David } \\
\text { Nabarro }\end{array}$ & & Springer Nature & & \\
\hline Reliefweb & & $\begin{array}{l}\text { SSRN } \\
\text { (Preprints) }\end{array}$ & & \\
\hline
\end{tabular}




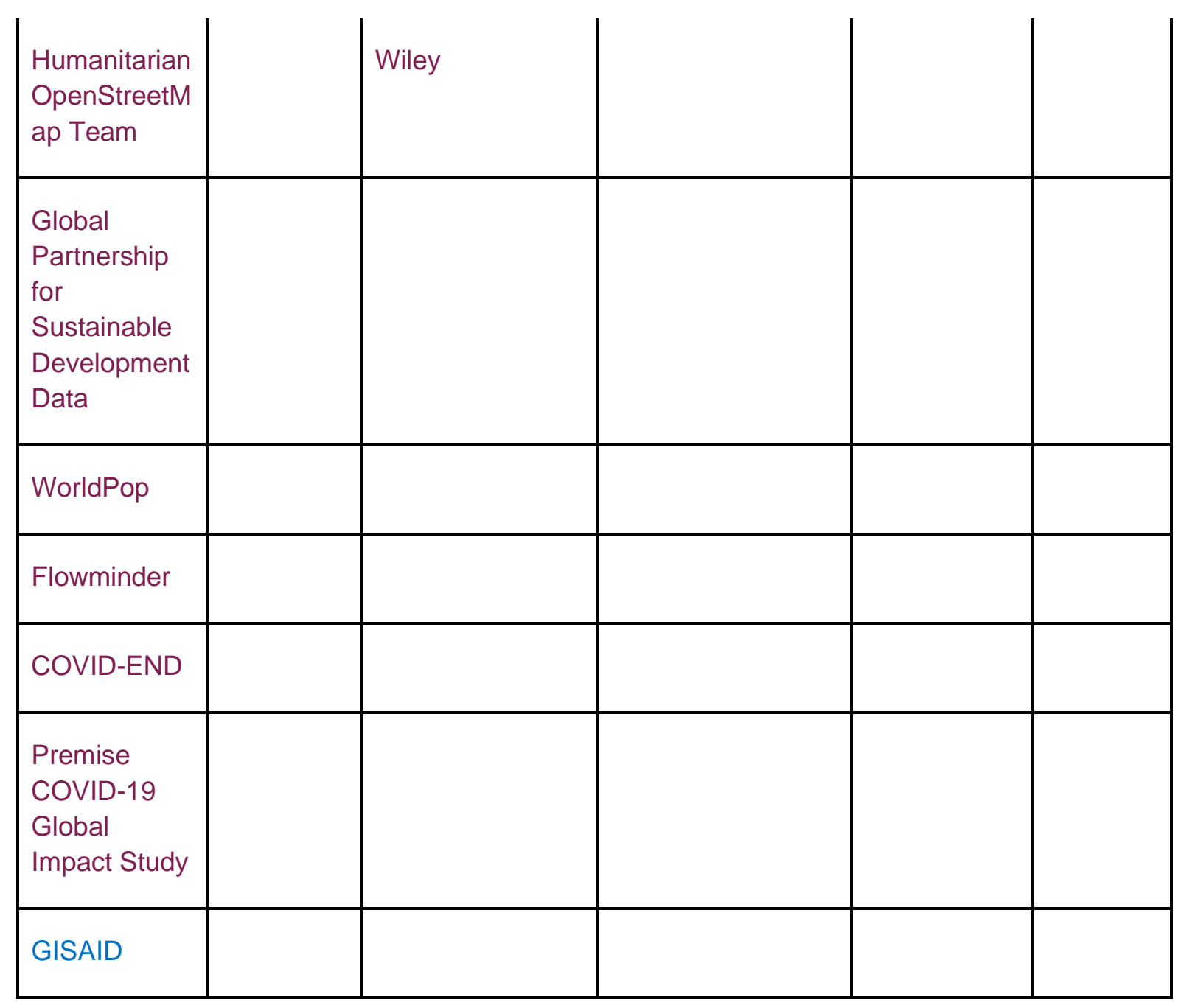




\section{Online learning \& events}

\begin{tabular}{|c|c|c|c|c|}
\hline Date & Title/URL & $\begin{array}{l}\text { Online } \\
\text { learning/event }\end{array}$ & Duration & Lead \\
\hline $\begin{array}{l}\text { February } \\
2021\end{array}$ & $\begin{array}{l}\text { COVID-19 } \\
\text { vaccination training } \\
\text { for health workers }\end{array}$ & Online training & $3 \mathrm{~h}$ & WHO \\
\hline 14.01 .2021 & $\begin{array}{l}\text { Evidence to impact in } \\
\text { crisis: how have we } \\
\text { measured up during } \\
\text { the COVID-19 } \\
\text { pandemic? }\end{array}$ & Webinar & $1 \mathrm{~h} 30$ & CGD \\
\hline 04.12 .2020 & $\begin{array}{l}\text { COVID-19, supply } \\
\text { chain resilience and } \\
\text { global trade }\end{array}$ & Webinar & $1 \mathrm{~h}$ & CGD \\
\hline 03.12 .2020 & $\begin{array}{l}\text { More money for } \\
\text { health services: What } \\
\text { is the tole of PFM in } \\
\text { the "new normal"? }\end{array}$ & $\begin{array}{l}\text { WHO \& CGD } \\
\text { Health systems } \\
\text { Governance \& } \\
\text { Financing }\end{array}$ & $1 \mathrm{~h} 30$ & Joe Kutzin \\
\hline 01.12 .2020 & $\begin{array}{l}\text { Solutions and support } \\
\text { for the mental } \\
\text { wellbeing of } \\
\text { community health } \\
\text { workers on the } \\
\text { COVID-19 frontline }\end{array}$ & Webinar & & $\begin{array}{l}\text { HSG TWG on CHWs } \\
\text { with The George } \\
\text { Institute for Global } \\
\text { Health }\end{array}$ \\
\hline 19.11 .2020 & $\begin{array}{l}\text { Looking at the } \\
\text { pandemic with a } \\
\text { gender lens }\end{array}$ & $\begin{array}{l}\text { Live Twitter } \\
\text { conversation }\end{array}$ & & SSHAP \\
\hline 16.11 .2020 & $\begin{array}{l}\text { HIFA and WHO } \\
\text { collaborate to } \\
\text { promote sharing of } \\
\text { experience and } \\
\text { expertise around the } \\
\text { maintenance of } \\
\text { essential health }\end{array}$ & $\begin{array}{l}\text { 4-week } \\
\text { discussion } \\
\text { starting } 16 \text { Nov }\end{array}$ & & HIFA \\
\hline
\end{tabular}




\begin{tabular}{|c|c|c|c|c|}
\hline & $\begin{array}{l}\text { services during (and } \\
\text { after) the pandemic }\end{array}$ & & & \\
\hline 10.11 .2020 & $\begin{array}{l}\text { COVID-19 vaccine } \\
\text { predictions part } 2 \text { : } \\
\text { estimating the time } \\
\text { before we approve } \\
\text { efficacious COVID-19 } \\
\text { vaccines }\end{array}$ & Online event & $1 \mathrm{~h} 30$ & CGD \\
\hline 16.10 .2020 & $\begin{array}{l}\text { Financing a Global } \\
\text { Public Health } \\
\text { Response }\end{array}$ & Online event & $1 \mathrm{~h} 30$ & CGD \\
\hline 02.10 .2020 & $\begin{array}{l}\text { Understanding and } \\
\text { Improving COVID-19 } \\
\text { Vaccine Portfolio }\end{array}$ & Online event & $1 \mathrm{~h} 30$ & CGD \\
\hline 21.09 .2020 & $\begin{array}{l}\text { Mitigating the } \\
\text { Economic and Health } \\
\text { Impact of COVID-19 } \\
\text { across Africa }\end{array}$ & Online event & $1 \mathrm{~h} 30$ & CGD, GF, AU \\
\hline June 2020 & $\begin{array}{l}\text { OpenWHO, the free, } \\
\text { open-access learning } \\
\text { platform for health } \\
\text { emergencies, now } \\
\text { offers } 10 \text { online } \\
\text { courses related to } \\
\text { COVID19. }\end{array}$ & Online courses & Varies & WHO \\
\hline $\begin{array}{l}\text { Available } \\
\text { now }\end{array}$ & $\begin{array}{l}\text { Standard } \\
\text { precautions: } \\
\text { Environmental } \\
\text { cleaning and } \\
\text { disinfection }\end{array}$ & Online course & 1 hour & WHO \\
\hline $\begin{array}{l}\text { Available } \\
\text { now }\end{array}$ & $\begin{array}{l}\text { COVID-19: Effective } \\
\text { Nursing in Times of } \\
\text { Crisis }\end{array}$ & Online course & $\begin{array}{l}2 \text { weeks - } \\
2 \text { hours } \\
\text { per week }\end{array}$ & $\begin{array}{l}\text { Johns Hopkins School } \\
\text { of Nursing }\end{array}$ \\
\hline $\begin{array}{l}\text { Available } \\
\text { now }\end{array}$ & $\begin{array}{l}\text { WHO Academy and } \\
\text { WHO Info mobile } \\
\text { applications }\end{array}$ & Mobile app & & WHO \\
\hline
\end{tabular}




\begin{tabular}{|c|c|c|c|c|}
\hline $\begin{array}{l}\text { Available } \\
\text { now }\end{array}$ & $\begin{array}{l}\text { COVID-19: } \\
\text { Pandemics, } \\
\text { Modelling and Policy }\end{array}$ & Online learning & $\begin{array}{l}2 \text { weeks | } \\
2 \text { hours } \\
\text { weekly } \\
\text { study }\end{array}$ & $\begin{array}{l}\text { FutureLearn UNESCO } \\
\text { UNITWIN Complex } \\
\text { Systems Digital } \\
\text { Campus/Open } \\
\text { University }\end{array}$ \\
\hline 11.5 .2020 & $\begin{array}{l}\text { COVID-19 Contact } \\
\text { Tracing course }\end{array}$ & Online learning & 5 hours & $\begin{array}{l}\text { Johns Hopkins } \\
\text { Bloomberg School of } \\
\text { Health }\end{array}$ \\
\hline $\begin{array}{l}7-28 \text { May } \\
2020\end{array}$ & $\begin{array}{l}\text { Virtual Evidence } \\
\text { Weeks }\end{array}$ & 5 sessions & $1 \mathrm{~h} 30$ & $\begin{array}{l}\text { International Initiative } \\
\text { for Impact Evaluation } \\
\text { (3ie) }\end{array}$ \\
\hline $\begin{array}{l}\text { Tuesdays } \\
\text { at } 1700 \\
\text { CEST } \\
\text { (Geneva } \\
\text { time) \& } \\
\text { Thursdays } \\
0830 \text { CEST } \\
\text { (Geneva } \\
\text { time) }\end{array}$ & $\begin{array}{l}\text { COVID-19 Open } \\
\text { online brief with Dr } \\
\text { David Nabarro }\end{array}$ & Event & $1 \mathrm{~h}$ & $4 S D$ \\
\hline $\begin{array}{l}\text { Available } \\
\text { now }\end{array}$ & $\begin{array}{l}\text { Emerging respiratory } \\
\text { viruses, including } \\
\text { COVID-19: methods } \\
\text { for detection, } \\
\text { prevention, response } \\
\text { and control }\end{array}$ & Online learning & 3 hours & WHO \\
\hline $\begin{array}{l}\text { Available } \\
\text { now }\end{array}$ & $\begin{array}{l}\text { Responding to } \\
\text { COVID-19: Real-time } \\
\text { training for the } \\
\text { coronavirus disease } \\
\text { outbreak }\end{array}$ & Online learning & $\begin{array}{l}\text { Multiple } \\
\text { self-paced } \\
\text { course }\end{array}$ & WHO \\
\hline $\begin{array}{l}25 \text { May } \\
2020\end{array}$ & $\begin{array}{l}\text { COVID-19: Tackling } \\
\text { the Novel } \\
\text { Coronavirus }\end{array}$ & Online learning & $\begin{array}{l}3 \text { weeks | } \\
4 \text { hours } \\
\text { weekly } \\
\text { study }\end{array}$ & $\begin{array}{l}\text { FutureLearn } \\
\text { LSHTM/UK PHRST }\end{array}$ \\
\hline
\end{tabular}




\begin{tabular}{|l|l|l|l|l|}
$\begin{array}{l}\text { Available } \\
\text { online now } \\
\text { without } \\
\text { mentors. } \\
\begin{array}{l}\text { Updated } \\
\text { version will } \\
\text { commence } \\
\text { early June } \\
2020\end{array}\end{array}$ & $\begin{array}{l}\text { COVID-19 } \\
\text { Diagnostics and } \\
\text { Testing }\end{array}$ & Online learning & $\begin{array}{l}3 \text { weeks } \\
3 \text { hours } \\
\text { weekly } \\
\text { study }\end{array}$ & $\begin{array}{l}\text { FutureLearn } \\
\text { FIND/LSHTM/ASLM }\end{array}$ \\
\hline $\begin{array}{l}\text { April 2020 } \\
\text { COVID-19 Critical } \\
\text { Care: Understanding } \\
\text { and Application }\end{array}$ & Online learning & $\begin{array}{l}5 \text { weeks } \\
1 \text { hour } \\
\text { weekly } \\
\text { study }\end{array}$ & $\begin{array}{l}\text { FutureLearn University } \\
\text { of Edinburgh \& Royal } \\
\text { College of Physicians } \\
\text { of Edinburgh }\end{array}$ \\
\hline $\begin{array}{l}\text { Available } \\
\text { now }\end{array}$ & $\begin{array}{l}\text { COVID-19 supporting } \\
\text { online courses }\end{array}$ & Online learning & $\begin{array}{l}\text { Multiple } \\
\text { self-paced } \\
\text { course }\end{array}$ & BMJ Learning \\
\hline
\end{tabular}

\section{Suggested citation}

Millington, K.A. and Reddin, S. (2021). COVID-19 Health Evidence Summary No.112. K4D

Evidence Summary. Brighton, UK: Institute of Development Studies. DOI:

10.19088/K4D.2021.021

\section{Rapid review methodology}

The rapid weekly search for peer-reviewed literature is carried out through a PubMed search with the following keywords ("COVID-19" OR "severe acute respiratory syndrome coronavirus 2" OR "2019-nCoV" OR "SARS-CoV2" OR "2019nCoV" OR "coronavirus" ) AND ("Africa" OR "South Asia" OR "Developing" OR "low-income" OR "low income" OR "lower-middle income" OR "low and middle income" OR "LMIC" OR "LIC" OR "global south") OR ("poverty") OR ("equity" OR "equities"), restricted to articles published in the previous 2 to 3 days, in English. This is complemented by a search of the homepage of the following high-impact global health journals: The Lancet journals, New England Journal of Medicine, Nature, JAMA, Annals of Internal Medicine, Cochrane Reviews, BMJ Global Health, the PLoS journals and a Twitter search of their Twitter pages. A search also of preprints from bioRxiv and medRxiv. Please note that papers that have not been peer-reviewed are highlighted in red. All primary research papers that relate to the primary and secondary impacts of the COVID-19 response in LMICs, and disease control and health system responses are included. Articles related to tackling the secondary impacts on other sectors are not included. Additional commentaries, opinions, and commissioned pieces are selected based on relevance.

The search for dashboards, guidelines, tools, editorials, comments, blogs, opinions and news is through the academic journals listed above, C19 resource hubs and following lead academics and professionals on Twitter. 


\section{About this report}

This weekly COVID-19 health evidence summary (HES) is based on 3.5 hours of desk-based research. The summary is not intended to be a comprehensive summary of available evidence on COVID-19 but aims to make original documents easily accessible to decision makers which, if relevant to them, they should go to before making decisions. The HES are not intended to replace medical or professional advice and the researcher or the K4D consortium cannot be held responsible for any decisions made about COVID-19 on the basis of the HES alone. K4D services are provided by a consortium of leading organisations working in international development, led by the Institute of Development Studies (IDS), with Education Development Trust, Itad, University of Leeds Nuffield Centre for International Health and Development, Liverpool School of Tropical Medicine (LSTM), University of Birmingham International Development Department (IDD) and the University of Manchester Humanitarian and Conflict Response Institute (HCRI).

This evidence summary was prepared for the UK Government's Foreign, Commonwealth and Development Office (FCDO) and its partners in support of pro-poor programmes. Except where otherwise stated, it is licensed for non-commercial purposes under the terms of the Open Government Licence v3.0. K4D cannot be held responsible for errors, omissions or any consequences arising from the use of information contained in this health evidence summary. Any views and opinions expressed do not necessarily reflect those of FCDO, K4D or any other contributing organisation.

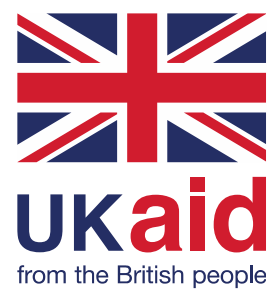

(c) Crown copyright 2021 